\title{
IT Project Environment Factors Affecting Requirements Analysis in Service Provisioning for the Greek Banking Sector
}

\author{
Krikor Maroukian \\ Printec Group of Companies, Athens, Greece. \\ Email: K.Maroukian@printecgroup.com
}

\begin{abstract}
The research undertaken within a Greek IT organisation specialising in service provisioning to the Greek banking sector discusses the various aspects of a number of identified environment factors within five distinct IT projects which affect the requirements analysis phase. Project Management (PMBOK ${ }^{\circledR}$ Guide $4^{\text {th }}$ ed.), IT Service Management (ITIL ${ }^{\circledR}$ v3) and Business Analysis (BABOK ${ }^{\circledR}$ Guide 2.0) framework practices applied to the various IT projects are highlighted in regard to improved activity execution. Project issue management, stakeholder management, time management, resources management, communication management and risk management aspects are presented. These are then linked to the identified environment factors so as to indicate the adaptability of an IT support team to changing environment factors in IT project environments and how the fulfilment of these factors can significantly contribute to effective requirements analysis and enhance the requirements management cycle.
\end{abstract}

Keywords: Requirements Analysis, Project Management, IT Service Management, Business Analysis, Greek Banking Sector

\section{Introduction}

International transaction systems have grown rapidly in the past decade, servicing more efficiently an ever growing number of debit and credit card holders throughout the world. Telecom Point-of-Sale (POS) devices offer an extended prism of services to cardholders in their daily purchase transactions. The Greek banking sector having sustained for many years the POS market, has now reached a high maturity level. This has enabled banking institutions to set a vision in terms of acquiring new technologies such as POS devices with embedded GPRS or Wi-Fi capabilities. It also means that suitable telecom network and IT infrastructure is established whereby POS management systems provide the required everyday service from banks to merchants and ultimately the cardholders. Requirements analysis is of significant importance in IT projects undertaken to support the POS management system environments that are currently installed at various banking institutions within Greece. As a consequence, it is the primary aim of this paper to investigate and identify the project environment factors that affect the requirements analysis phase in the Greek banking sector, through a series of five IT projects with distinct characteristics each, carried out by a POS management systems Support Team acting as part of Printec Group's Software R \& D Division.

\subsection{Background}

The research paper focuses on the various issues faced in the requirements analysis during the service design, service transition and service operation execution of five large to medium-scale IT projects undertaken within the Greek banking sector. The primary deliverable of all projects was the deployment of a POS or Terminal Management System (TMS) within five Greek banks, three of them forming part of the four largest banking establishments within the Greek banking market. Services are the means of delivering value to customers by facilitating outcomes customers want to achieve, without the ownership of specific costs and risks [1]. Moreover, there is extensive reference on the various methodologies and practices employed towards client requirements identification, categorisation and dissemination of information to other corporate teams and the effect of this analysis on project deliverables. The paper also discusses the established practices which aided the overall process of re- 
quirements management. Four teams were highly involved in the IT projects undertaken. These consisted of the TMS implementation and support team, the TMS development team of Printec Group Software R\&D Division and the Printec Greece e-Payments Department teams of POS application development and POS Helpdesk; a subsidiary of Printec Group. Teams from Printec Group reported to the Steering Committee and both had an individual appointed at a managerial role. The Steering Committee consisted of the Software R\&D Division Director and the Group General Manager. The Printec Greece e-Payment Department POS Helpdesk and POS Development teams were headed by the Technical Supervisor and the Head of Software Engineers.

\subsection{Project Management Plans}

The aim of IT projects undertaken was to install or upgrade the TMS software for five banking institutions. An eighteen month period covered the entire duration of the five executed IT projects starting from May 2008 and lasting till November 2009, see Table 1. Notice that all project implementations refer to system upgrades except for Sigma Bank which resulted to a fresh installation of TMS at Printec Greece premises.

Printec Group's IT service provisioning to the five banking institutions can be further divided into areas such as POS management, merchant management, POS issue management, reports management and client specific business needs. In addition, the client's point of contact with Printec Group's internal software development department was the TMS support team. Therefore, the TMS implementation and support team was responsible for highlighting or escalating any issues or requirements that might arise regarding client needs. Soon it became apparent that healthy relationships between the client and the IT services supplier when maintained, through good professional practices such as regular communication updates on current issues faced, post-visit reporting on decisions reached, issue response and resolution or simply updates on a new product release, can result in higher client utility and improved practice in the requirements capture activity.

\section{Project Management, Service Management and Business Analysis Frameworks for Requirements Analysis in the Greek Banking Sector}

From the very beginning of the IT projects, it was realised that a defined period for requirements identification cycle would ensure that all client requirements would be recorded as part of the documentation practices already established within Printec Group. These would be taken into full consideration by all involved stakeholders for the release of the new TMS software [2]. Identification of business and user requirements was directly related to functional and non-functional requirements. Functional requirements relate to the scope of work or functionality the software must have whereas non-functional requirements refer to look and feel, usability, performance, security and maintainability and support requirements of the software [3].

Moreover, maintaining an up to date set of requirements gained significant priority and became a crucial aspect of applied IT project management practices. It was important that all stakeholders of the TMS support and software development teams had a perfectly aligned perception of what the client required so as to avoid a misconception of expressed client requirements.

\subsection{Applied Requirements Analysis and Business Analysis Practices and Methodologies}

The requirements analysis process refers mainly to recorded and accepted requirements that will form part of project deliverables. The acceptance stage can be con-

Table 1. Project management plans duration for five IT projects in the Greek banking sector.

\begin{tabular}{lcccc}
\hline \multicolumn{1}{c}{ Bank } & Project Start Date & $\begin{array}{c}\text { Project End } \\
\text { Date }\end{array}$ & $\begin{array}{c}\text { Service Delivery } \\
\text { Duration (month) }\end{array}$ & TMS version \\
\hline Beta Bank & $5 / 5 / 2008$ & $14 / 02 / 2009$ & $91 / 4$ & Upgrade TMS6 $\rightarrow$ TMS7 \\
Gamma Bank & $7 / 7 / 2008$ & $18 / 12 / 2008$ & 5 & Upgrade TMS7 $\rightarrow$ New version of TMS7 \\
Delta Bank & $7 / 11 / 2008-25 / 11 / 2008 \&$ & $25 / 8 / 2009$ & $3^{3 / 4}$ & Upgrade TMS7 $\rightarrow$ New version of TMS7 \\
Sigma Bank & $20 / 05 / 2009-25 / 8 / 2009$ & $11 / 05 / 2009$ & $3 / 4$ & Install TMS7 \\
Omega Bank & $23 / 4 / 2009$ & $24 / 11 / 2009$ & 6 & Upgrade TMS6 $\rightarrow$ TMS 8 \\
\hline
\end{tabular}


ducted under the auspices of a formal meeting with the client during which essential business requirements are discussed, recorded and timelines of service delivery provided to the client by senior management. Later on, the agreed requirements to be implemented and timelines should be communicated to all project stakeholders so that organisational efforts for the release of the new TMS software are aligned according to specifications. In effect the purpose of the requirements analysis process is to establish which requirements have been identified, the case of whether there needs to be provided clarification in regard to a requirement and finally accept the implementation of the necessary software development to release the new software product which would fully conform to client requirements. Three processes were identified throughout the requirements analysis process as the key ingredients to best practice. These are the Requirements Identification, Requirements Categorisation and Requirements Prioritisation. It was made clear that there was a set of requirements to which the client agreed upon a formal meeting with Printec's senior management and that there was flexibility in new requirements to be accepted after a new release had been scheduled and implemented in terms of establishing an independent project altogether to implement these new requests.

Firstly requirements capturing or identification procedures were established whereby client requirements were identified as follows:

1) Senior management buy-in for the project indicated strong commitment from Printec Group's side to the requirements analysis process;

2) Enquiries and incident logging through the Service Desk;

3) Frequent formal visits to the bank's site;

4) The use of a coherent vocabulary or common glossary among Printec Group and bank employees was instrumental towards an improved understanding of requirements identification;

5) A requirements identification period resulted to improved requirements capture and a better understanding of the software functionality the client was expecting to receive.

Secondly the requirements categorisation process refers to identifying the software component or module to which a requirement refers. There were two types of requirements in regard to resource allocation to tasks for their implementation. Those that could be handled by the support team and those that had to be escalated to the software development team. Resource allocation to tasks was managed through the issue management system. In addition, requirements categorisation can be carried out having in mind functional and non-functional require- ments. Functional requirements describe the functionality of a system. These are sometimes known as software capabilities. On the other hand, non-functional requirements act to constrain the solution and can be referred to as constraints or quality requirements. In fact, non-functional requirements can be further classified according to whether they are performance requirements, maintainability requirements, security requirements or reliability requirements.

In addition to categorisation of requirements the established issue management system within Printec Group assisted in the prioritisation of what requirements required immediate implementation in the new software release and which ones could be implemented in a later software release. Within Printec Group the following points were considered important prioritisation criteria:

1) Importance of requirement to client satisfaction. In effect this is what the client expressed as necessary to sign off user acceptance testing and eventually project implementation;

2) Criticality of requirement in client production environment; This attributes to the severity of implications caused in the client production level if the requirement was not implemented;

3) Capability of re-tracing previously documented requirements for re-use;

4) Resource allocation to requirements tasks;

5) Cost of implementation per requirement.

Note that there are certain challenges associated with requirements prioritisation which need to be carefully considered as defined in $\mathrm{BABOK}^{\circledR}[4]$ :

- Non-negotiable demands whereby the client is unwilling to commit to any trade-offs and ranks all requirements as high priority;

- Unrealistic tradeoffs whereby the service provider's solution development team may intentionally or unintentionally try to influence the result of the prioritisation process by overestimating the requirements implementation complexity.

Subsection 2.2 refers to project management practices established for Beta Bank, Gamma Bank, Delta Bank and Omega Bank. In the Sigma Bank case, the installation of the TMS software was outsourced. Therefore TMS was deployed at the Printec Greece premises thus mitigating the execution of the requirements analysis process to the Printec Greece-e-Payments Department POS Helpdesk team which in turn collaborated with Sigma Bank - Cards Division officials. The mental mismatch model can be also considered for the Sigma Bank TMS deployment project, see Subsection 3.1.4, in a slightly different stakeholders' context but always indicating the necessity to cater for misconception of expressed client requirements 
between various parties and teams as shown in Figure 1.

\subsection{Applied Project Management and Service Management Practices and Methodologies}

The TMS implementation and support team performance was constantly measured through Key Performance Indicators (KPI) which indicated that most of the time all issues were resolved and whenever serious issues arose with high business impact these were escalated to the TMS development team to address the issue in software development terms. Some of the frequently monitored and reported KPIs include, issues closed at first call at the Service Desk, issues closed after second level support consultation, average time per call per analyst, issues escalated for third level support to TMS software development and POS software applications development teams. In effect, there was usually a small backlog of client issues relating to TMS installations which made it possible to counteract more effectively to newly reported issues.

Throughout the duration of the IT project the senior management style was consultative whereby decisions were taken by seeking the opinions and views of the teams prior to a decision being made [5]. However final decisions lied solely in the judgment of the Steering Committee. Furthermore, knowledge transfer on TMS usability matters from the development team to the support team was vital. This was conducted through formal and informal meetings, online material and communication, computer based training (CBT) and constant involvement to client issue resolution.

An established Service Desk operating according to ITIL specifications, was utilised to control and monitor TMS support performance levels. In particular, it was decided who would take responsibility of escalating issues to the development team, aligning client site TMS software items with Printec Group's TMS related configuration items (CI) and who would trigger and organise formal team meetings. Moreover, work delegation was essential in assigning tasks to resources by taking into consideration resource availability and the individual's expertise on the different functionality aspects of TMS. In this way incident response timeframes to the client would be minimised. Performance reporting to the Steering Committee was essential. This involves collection and distribution of performance information to project stakeholders [6].

The established flow of information and client requirements identification to escalation management required the TMS Support team members be at the heart of the process as depicted in Figure 2. Notice that the Steering Committee and Software Development team, at

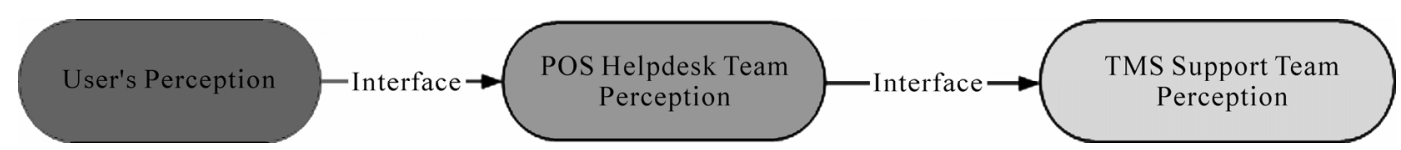

Figure 1. The mental model mismatch for the Sigma Bank-TMS deployment project.

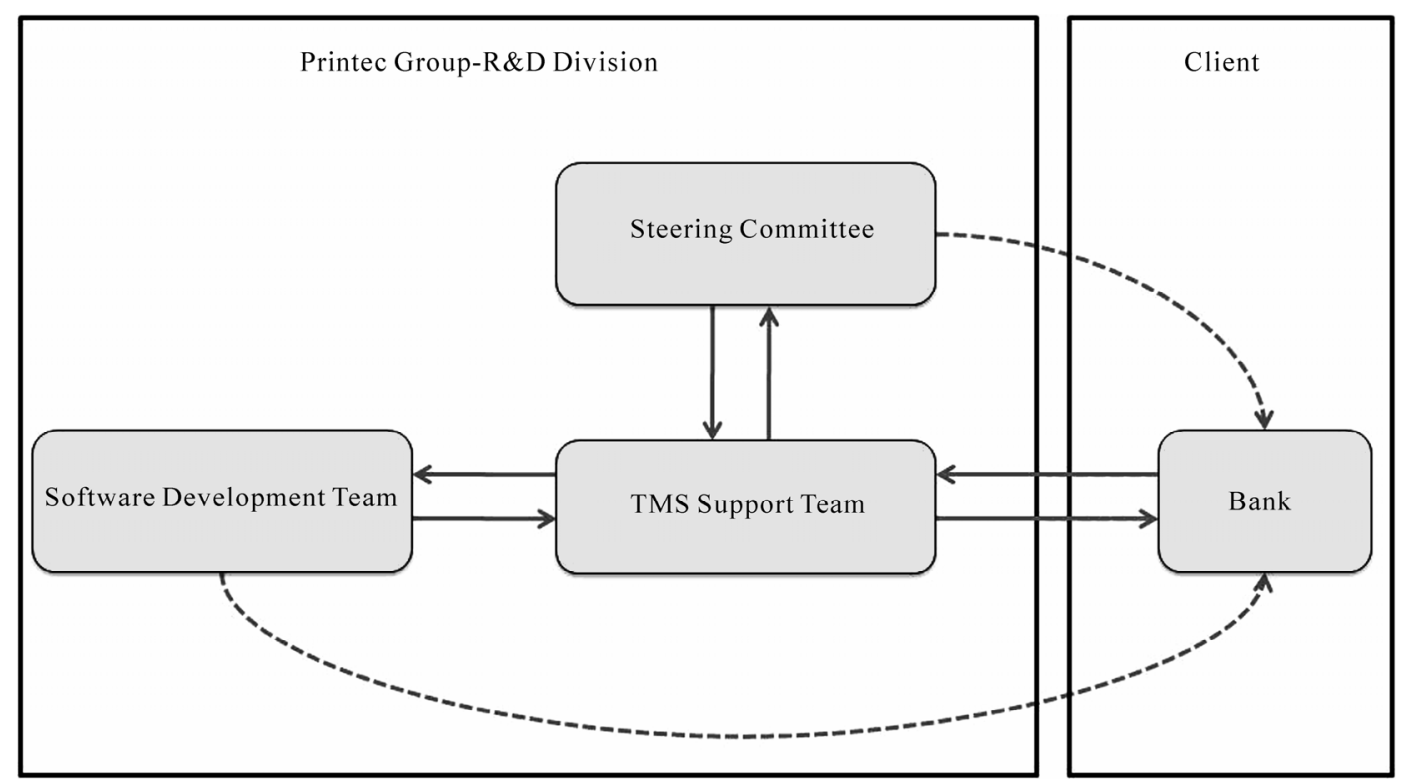

Figure 2. Printec Group-Software R \& D Division communications mapping with clients. 
exceptional cases, had to be involved in the IT support process with the client. This occurred due to the fact that certain requirements had to be clarified in technical terms to avoid ambiguity so as implementation and the end result was exactly what the client requested initially. In addition, this also occurred in situations when senior management had to engage in the decision making process with the client or even to schedule high profile meetings. In fact, decisions or agreements reached in collaboration with Printec Group's senior management would indicate higher commitment to the TMS project from the client side.

\subsubsection{Established Service Desk}

The implementation of the Service Desk for the TMS support team entailed the recording of landline incoming/outgoing telephone communications. This was achieved by monitoring and responding to all outstanding activities/requests/complaints, creating and maintaining a Known Error Database (KED) and reporting on a monthly basis to the Software R \& D Division Director on TMS support productivity and efficiency.

The established Service Desk within the TMS support team served as a technique for capturing and recording client requirements

\subsubsection{Issue Reporting and Statistics Provision}

A suitable frequency of reporting and review was established, depending upon the importance of the review. Providing results in graphical form is useful for presenting management overviews on major areas of interest [7]. To provide a common service objective, it was important that all TMS stakeholders were aware of major issues, concerns, performance levels and achievements of the entire Software R \& D Division and not just their team. Table 2 below, shows the monthly performance statistics produced for the Director of the Software R \& D Division by the owner of the TMS Support Service Desk.

\section{Investigation of Environment Factors-Five Case Studies in the Greek Banking Sector}

This chapter presents a discussion on each of the five IT projects the Printec Group TMS support team embarked on and unravels the factors that affected the requirements analysis phase which in turn resulted in delays or improvements to project timeframes. The case studies are presented in time sequence as they occurred throughout an eighteen month period.

The requirements analysis and business analysis (BA$\mathrm{BOK}^{\circledR}$ ) procedures implemented by the TMS support team have been previously described see Subsection 2.1. Project Management $\left(\mathrm{PMBOK}^{\circledR}\right)$ and IT Service Management (ITIL ${ }^{\circledR}$ v3) framework practices have been thoroughly described in Subsection 2.2.

\subsection{Terminal Management System Deployment Projects for Five Banking Institutions within Greece}

Each project presented in this section carries a different set of characteristics which distinguishes it from the rest in terms of size of client organisation, applied corporate

Table 2. Monthly performance statistics of the TMS support service desk.

\begin{tabular}{lcccc}
\hline & Total issues & \% Change & Resolved issues & \% \\
\hline September 2008 & 25 & $0.00 \%$ & 24 & $96.00 \%$ \\
October 2008 & 27 & $7.41 \%$ & 24 & $88.89 \%$ \\
November 2008 & 30 & $10.00 \%$ & 29 & $96.67 \%$ \\
December 2008 & 30 & $0.00 \%$ & 28 & $93.33 \%$ \\
February 2009 & 29 & $-3.45 \%$ & 28 & $96.55 \%$ \\
March 2009 & 28 & $-3.57 \%$ & 26 & $92.86 \%$ \\
May 2009 & 27 & $-3.70 \%$ & 27 & $100.00 \%$ \\
Jun-Jul 2009 & 13 & $-107.69 \%$ & 13 & $100.00 \%$ \\
Aug - Sep 2009 & 19 & $31.58 \%$ & 18 & $94.74 \%$ \\
Sep- Oct 2009 & 17 & $-11.76 \%$ & 16 & $94.12 \%$ \\
Nov 2009 & 15 & $-13.33 \%$ & 14 & $93.33 \%$ \\
\hline
\end{tabular}


IT policies, IT resources and human resources availability, project complexity, issue management, demand management, scope management, time management and financial management (project budget). The projects undertaken for the Greek banking sector are presented below in chronological order, see Table 3. It is clear that the project engaged with the largest banking institution, Beta Bank, faced the highest number of issues requiring resolution. In the ITIL context these can be classified as incidents or problems. In case problems arose the appropriate changes were requested and upon approval of the Change Advisory Board (CAB) a new TMS release was scheduled with a problem resolution patch. Moreover, the organisation criticality category describes the vitality and business operations impact each customer represents to Printec Group as a business customer. For example, Table 3 shows that Beta Bank is considered to be a strategic customer of Printec Group and therefore any incidents or problems arising from any Beta Bank project require additional attention in accordance to the customer specific Service Level Agreement (SLA). Project complexity refers to the level of bureaucratic processes put in place by the customer, customer decision making time and processes on expression of customer requirements, time of response to service provider requests and in general elements that might materialise risks which will translate, as a consequence, in terms of project delays. Lastly, the number of tasks assigned per project was similar in all five case studies as shown in Table 4.

Note that throughout the duration of the five case studies a project manager was working in conjunction with the TMS implementation and support team so as both the service provider and the customer complied with the agreed project plan and project schedule. The assignment of tasks to individuals supported the need of task ownership. In this way individual responsibility for the completion of tasks on-time and within project scope was encouraged.

\subsubsection{Beta Bank}

The first project for the deployment of TMS at the Beta Bank premises, the largest banking institution within South-Eastern Europe, started in May 2008 and lasted till February 2009. With an approximate duration of nine months and thirty-six reported issues, see Figure 3, it was the largest and most complex project of all. High

Table 3. TMS deployment project characteristics for five banking institutions within Greece.

\begin{tabular}{lccccc}
\hline Bank & $\begin{array}{c}\text { Completion } \\
\text { Duration (months) }\end{array}$ & Project Issues & $\begin{array}{c}\text { Organisational } \\
\text { Criticality }\end{array}$ & $\begin{array}{c}\text { Project } \\
\text { Complexity }\end{array}$ & $\begin{array}{c}\text { \% of Tasks } \\
\text { Completed }\end{array}$ \\
\hline Beta Bank & 9 & 36 & High & High & $100 \%$ \\
Gamma Bank & 5 & 16 & Medium & Medium & $100 \%$ \\
Delta Bank & $33 / 4$ & 11 & Medium & Medium & $100 \%$ \\
Sigma Bank & $3 / 4$ & 6 & Low & Low & $100 \%$ \\
Omega Bank & 6 & 11 & Medium & High & $100 \%$ \\
\hline
\end{tabular}

Table 4. A standard TMS deployment project schedule.

\begin{tabular}{lcc}
\multicolumn{1}{c}{ Task } & $\begin{array}{c}\text { Completion } \\
\text { Duration (days) }\end{array}$ & Owner \\
\hline Project kick-off meeting & 0 & Software Support Engineers, Project Manager \\
Requirements Analysis & 5 & Software Support Engineers, Project Manager \\
$\begin{array}{l}\text { Data migration from old to new system } \\
\text { Setup of User Acceptance Testing (UAT) } \\
\text { environment at client site }\end{array}$ & 15 & Software Support Engineer 1 \\
$\begin{array}{l}\text { Testing at client site } \\
\text { UAT sign-off and software activation in } \\
\text { production }\end{array}$ & 15 & Software Support Engineer 2 \\
\hline
\end{tabular}




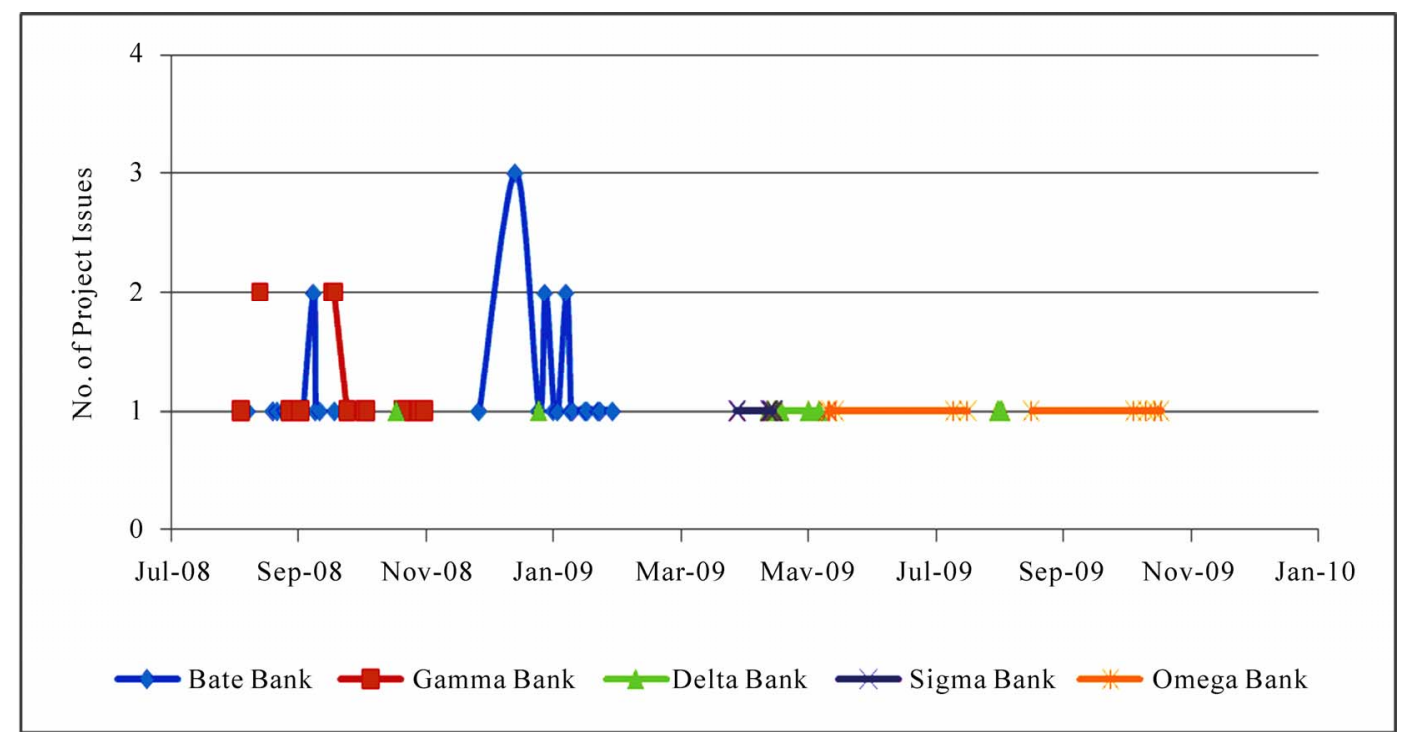

Figure 3. Recorded issue rate per day throughout the duration of all five projects.

project complexity resulting to project timeframe delays existed due to several reasons as described below:

1) High issue reporting rates, see Table 3 , meant that TMS support and TMS Development team members had to first resolve the issues so as to progress through the project management plan;

2) Numerous change requests to project requirements and thus deliverables during the User Acceptance Testing stage. In general, software development at this stage entails high costs;

3) Numerous confirmation requests for undocumented TMS workflow which meant that Printec Group's Intellectual Property had to be preserved while satisfying client requests;

4) TMS administrators were constantly seeking reassurance on system management matters;

5) Constant requests for additional training provision;

6) The large size of corporate POS Helpdesk and POS Faults Departments resulted in scheduling requests for additional training sessions;

7) Beta Bank being a high-profile client meant that no trade-offs could be made during the requirements analysis phase. Without any ground to negotiate identified requirements implementation there is additional risk on the service supplier side during service delivery.

In addition, the prolonged requirements analysis period, at the beginning of the project, was beneficial in the sense that certain requirements were well defined and a good understanding of their implementation was acquired. However, the prolonged period of service design, service transition and service delivery in general, had a serious impact on project constraints e.g. extended pro- ject duration and contributed to higher project complexity as well.

\subsubsection{Gamma Bank}

The second project for the deployment of TMS at Gamma Bank premises, the third largest Greek banking institution within Greece, started in July 2008 and lasted till December 2008. With an approximate duration of five months and sixteen reported issues, see Figure 3, it was the third largest and complex project of all. Medium project complexity resulting to project timeframe delays existed due to the following reasons:

1) Established IT security corporate policies meant that several authorisations were required to conduct simple activities such as the installation of new software on UAT and production environments or the retrieval of a database backup file. Usually this resulted in task delays;

2) Whenever task delays occurred throughout the project lifecycle, the rate of visits increased so as to ensure that TMS deployment work was carried out as planned.

Gamma Bank having sustained a highly sophisticated and secure TMS IT environment on its premises, assisted in keeping the project complexity at a medium level compared to the rest of the projects even though high IT security levels resulted at some cases to project timeframe delays. As observed, in the case of Beta Bank, when nearing TMS upgrade dates the recorded issues to be resolved experienced an increased rate. As a consequence the Printec Group TMS Support team had to be highly responsive, during these periods, regarding requests communicated by the banking institution's management. In fact, this team behaviour had to be consistent within all client project environments. 


\subsubsection{Delta Bank}

The third project for the deployment of TMS at Delta Bank premises, subsidiary to the largest worldwide financial institution based in the USA, started in November 2008 and lasted till August 2009 with a halting period of six months due to events caused by the recent global economic recession. With an approximate duration of three months, three weeks and eleven reported issues, see Figure 3, it was the fourth in scale project of all with medium complexity. Medium project complexity resulting to project timeframe delays existed due to certain reasons as described below:

1) Established IT security corporate policies meant that several authorisations were required to conduct simple activities such as the installation of new software on UAT and production environments or the retrieval of a database backup file. Usually this resulted in task delays;

2) Whenever task delays occurred throughout the project lifecycle, the rate of visits increased so as to ensure that TMS deployment work was carried out as planned;

3) The TMS deployment project timeframe of delivery was largely affected by the recent economic turmoil. Even though the active project duration was recorded as three months and three weeks; adding the inactive period to the project duration equals to nine and a half months.

The Delta Bank TMS environment IT setup shared a lot of similarities to that of Gamma Bank since both were seeking upgrades of older TMS7 systems to the most recent TMS7 software releases. Therefore, the major factors which affected requirements analysis and project completion are stated along the same lines.

\subsubsection{Sigma Bank}

The fourth project for the deployment of TMS on behalf of Sigma Bank at Printec Greece premises, a small banking institution based in Greece, started at the end of April 2009 and lasted till mid-May 2009. With an approximate duration of three weeks and only six reported issues, see Figure 3, in terms of scope, time and budget, as stated in $\mathrm{PMBOK}^{\circledR}$, this was a successful project. Low project complexity resulted to the effective application of project management and service management practices and an improved project timeframe for reasons described below:

1) High control of outsourced TMS deployment service owned by Printec Greece e-Payments Department POS Helpdesk team;

2) A quick issue resolution process in collaboration with Printec Greece e-Payments Department;

3) IT security policies were set internally by Printec Group TMS support and Printec Greece e-Payments Department POS Helpdesk teams.
Sigma Bank on its own involved a fresh installation of TMS at the premises of Printec Greece; the Greek subsidiary of Printec Group. Requirements definition and management was conducted in an organised and concise manner, from the beginning of the project. The materialisation of a TMS6 system failure meant that reactive tasks had to be put in place for urgent issue resolution purposes. All tasks falling within the data migration and installation barriers were executed in a timely and highly responsive manner. Furthermore, good communications among project stakeholders throughout the duration of the project was executed. This involved communication of the TMS support team with counterparts in the Printec Greece e-Payments Department. The Technical Supervisor of the Printec Greece e-Payments Department POS Helpdesk team was appointed the TMS administrator. Regarding risk management techniques risk owners were appointed for the data migration, installation, maintenance and administration tasks. As a result emerging issues were resolved within reasonable timeframes. A request was made for risk control and monitoring purposes so that tasks were put in place to avoid future risk materialisation e.g. establish daily TMS system database backup plan.

\subsubsection{Omega Bank}

The fifth project for the deployment of TMS at Omega Bank premises, the Greek subsidiary of a French financial services Group, started in May 2009 and lasted till November 2009. With an approximate duration of six months and eleven reported issues, see Figure 3, it was the second largest and most complex project of all. High project complexity resulting to project timeframe delays existed due to several reasons as described below:

1) Low availability of IT resources;

2) Insufficient IT infrastructure capabilities;

3) Inexistent corporate IT security policy;

4) Lack of appointment of TMS administrator(s);

5) As a result additional TMS performance issues were recorded due to compliance failure of the client to minimum system specifications;

6) An increased rate of visits so as to ensure that TMS deployment work was carried out as planned meant that the project timeframe suffered additional delays;

7) Numerous training sessions had to be organised due to an apparent indifference of Omega Bank POS Helpdesk staff to acquire the knowledge necessary to proceed to service delivery;

8) The project was part of the first live deployment of new corporate TMS software product during which, the TMS support team underwent a knowledge transfer and knowledge acquisition period as part of the service tran- 
sition process.

During the project initiation stage Omega Bank seemed to have many similarities to the Beta Bank TMS project environment. However, a known risk materialised over the course of the project which regarded low IT resources support capability from the client side. This meant that most of IT resources management had to be conducted by the Printec Group TMS Support team which increased the responsibility and accountability factors on the side of the service supplier. Although the initial project plan presented to Omega Bank did not include this kind of additional tasks the overall project duration was largely affected. Moreover, the inexistence of an appointed TMS administrator added to the already high complexity level of the project. However, due to the fact that the newly released TMS8 software was to upgrade the old TMS6 system project completion was of high priority and significance and therefore the efforts focused in enabling a smooth transition for the day-today Omega Bank POS management activities.

\subsection{Identified Environment Factors Affecting Requirements Analysis}

In Subsections 3.1.1 through 3.1.5, a series of IT projects has been presented each with its own distinct characteristics. The five project environments highlighted in terms of project management, service management and business analysis distinguish themselves though certain identified influential factors. These factors relate to three domains. Firstly, factors relating to human behaviour and competencies which entails individual task ownership, responsibility, accountability, competence and skills. Secondly, factors related to policy and standards compliance and lastly factors influenced by IT systems architecture and IT resources availability.

The total project duration recorded at the end of each project lifecycle indicated a correlation between the organisation criticality, see Table 3, and total project issues per project, see Figure 4, to form a similar pattern as shown in Figure 5.

Throughout the lifecycle of a project and from information recorded during project closure in the lessons learned documentation, certain environment factors were identified which carry a major influence on service delivery time and budget management as well as requirements analysis and implementation acceptance by the client. These factors are as follows:

1) IT security policies and standards applied, e.g. ISO/IEC 27000;

2) Corporate software deployment policy procedures on the client side;

3) Software documentation provision e.g. user manual,

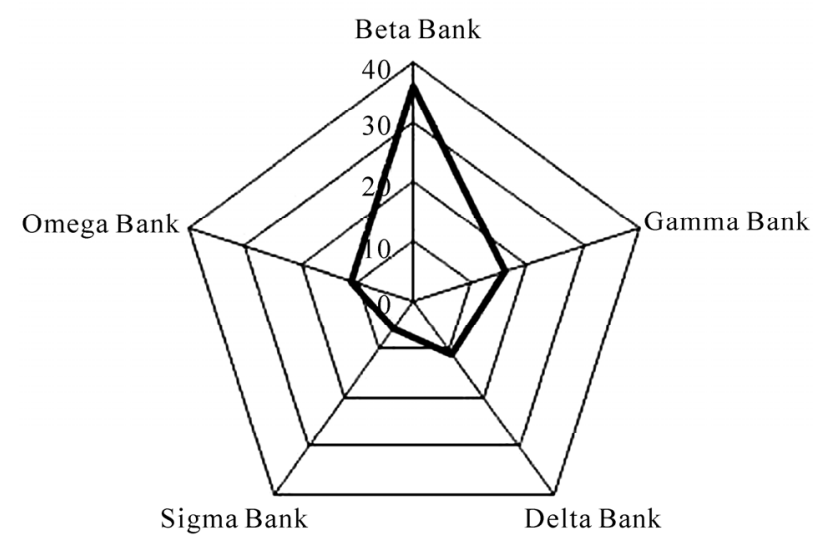

Figure 4. Total project issues per project depicted on a radar chart.

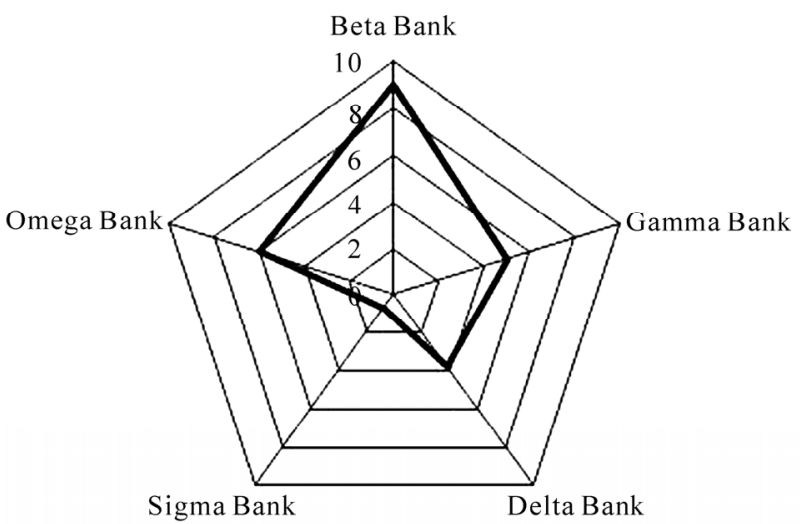

Figure 5. Total project duration (months) per project depicted on a radar chart.

operator manual, etc.;

4) Rate of software training sessions for system administrators and system users;

5) Rate of formal walkthroughs, meetings and visits at the client site;

6) Appropriate IT support team skills on the service provider side;

7) System administrator(s) attitude(s) in terms of new requirements requests made, commitment to software administration, comprehension skills of new software through training sessions and user acceptance testing sign-off willingness;

8) IT resources availability i.e. hardware, servers, etc.;

9) Senior management commitment from both, the service supplier and the client;

10) Selection of appropriate deployment period. For example, festive periods which result in high transaction rates, summer time when seasonal shops operate e.g. touristic shops, restaurants, hotels, should be carefully considered when making request for change for TMS; 
11) Definition of project stakeholders' task ownership at the beginning of the project, for both the service supplier and the client, using techniques such as a RACI (Responsible-Accountable-Consulted-Informed) chart.

The aforementioned factors should not be assigned to a weighting system whereby each factor is categorised according to impact on project deliverables. The reason behind this being that throughout the duration of the five case studies presented wherever one or more of these factors was not satisfied there were bound to be user acceptance issues and system deployment delays. The question at hand is how a service supplier can satisfy as many of the factors stated previously, in order to fulfil client requirements and attain high client satisfaction.

Sigma Bank forms a distinct exception to the above identified factors due to the fact that it signifies an outsourcing IT project i.e. the TMS software was installed internally at Printec Greece premises. In this case, the control over the IT competency of POS management system was given exclusively to Printec Greece-POS Helpdesk team. Therefore, certain environment factors were considered unnecessary. For example, as mentioned earlier the Sigma Bank TMS7 installation was a result of an unexpected system failure and urgency emerged for the issue to be resolved as soon as possible. Therefore the factor regarding suitable yearly period deployment cannot be applied in this case since the system recovery and deployment processes commenced as soon as the system failure occurred.

\section{Findings}

A number of noteworthy findings are evident regarding the service provision of a Terminal Management System, developed from Printec Group-R\&D Division, to five banking establishments operating within Greece. Moreover, a full cycle of requirements analysis has been described whereby requirements identification, categorisation and prioritisation processes combined with best practice in Project Management according to $\mathrm{PMBOK}^{\circledR}$, IT Service Management according to ITIL ${ }^{\mathbb{B}}$ and Business Analysis according to $\mathrm{BABOK}^{\circledR}$ can be instrumental towards successful requirements analysis.

The identification of eleven factors that have potentially influenced the five project environments of Beta Bank, Gamma Bank, Delta Bank, Sigma Bank and Omega Bank indicates the necessity to cater for each one in order to fulfil successfully client requirements in the Greek banking sector. In other words, to increase project implementation success rates these factors should be considered thoroughly. If success is to be measured in terms of Scope, Time and Budget as stated in $\mathrm{PMBOK}^{\circledR}$, then that means that projects should be governed by generally accepted best practice frameworks. For the five case studies presented in Section 3, the project completion rate and projects within scope rate was $100 \%$. In general, these were caused by the project environment factors identified in Subsection 3.2. There was no case of forceful project closure even though on one occasion there was a halting period which lasted up to six months as a consequence of the effects of the current economic recession on Delta Bank. It is also notable, that the Sigma Bank project signified an outsourced IT competency to Printec Greece. The TMS deployment project was a success in project management, service management and requirements analysis terms but this does not necessarily mean that outsourcing is an option for any client of any size. Sigma Bank holds a relatively small corporate structure, the lowest number of POS devices in its TMS database and therefore the lowest number of requirements.

It has been shown that constantly employed requirements analysis tools, techniques and methodologies as well as issue management, time management, resources management, risk management, stakeholder management and service supplier Service Desk Operations reporting, assist greatly in the process of capturing client requirements in a timely fashion. The need of senior management buy-in in the process of requirements identification has been also stressed. This increases client confidence and maintains a healthy client-supplier relationship. Requirements categorisation has revealed that when prioritising requirements, influencing factors should be mainly customer centric.

Finally a thorough account of PMBOK ${ }^{\circledR}$, ITIL ${ }^{\circledR} \mathrm{v} 3$ and $\mathrm{BABOK}^{\circledR}$ methodologies has been given whereby scope management, time management, resources management, issue management, Service Desk, availability management, stakeholder management, risk management, business analysis and release management processes have been blended with requirements analysis tasks in order to maximise business benefits. As PMBOK ${ }^{\circledR}$, ITIL ${ }^{\circledR} \mathrm{v} 3$ and $\mathrm{BABOK}^{\circledR}$ methodologies are used to a certain extent, though limited within Greece, this research has indicated the significant opportunities presented for improved requirements analysis when the $\mathrm{PMBOK}^{\circledR}, \mathrm{ITIL}^{\circledR} \mathrm{v} 3$ and BABOK $^{\circledR}$ frameworks are used in conjunction in IT project environments for the Greek banking sector.

\section{Conclusions}

This paper has presented the various environment factors with either negative or positive impact on the requirements analysis phase while undertaking five IT projects with differing environmental setups within the Greek banking sector. The essence of keeping healthy cli- 
ent-supplier relationships, appropriate IT and human resource provisioning by the customer side, appropriate visit frequency rates at the customer site, provision of high-quality software documentation from the service supplier side, training provision of system administrators and users from the service supplier, compliancy to corporate IT security and software deployment standards at the client site, setting service transition milestones in accordance to specific yearly periods, service supplier and client senior management commitment to project deliverables as well as the use of PMBOK ${ }^{\circledR}$, ITIL ${ }^{\circledR}$ and $\mathrm{BABOK}^{\circledR}$ methodologies has been highlighted. Vital issue management tools and techniques have also been presented such as the importance of keeping an up-todate set of recorded issues as part of Service Desk operations and issue resolution statistics reporting to the Director of Printec Group-Software R \& D Division. Moreover, the effects of proper guidance in the requirements analysis process which entails the requirements identification, categorisation, prioritisation, implementation and release stages has been clearly indicated. The specific PMBOK $^{\circledR}$, ITIL $^{\circledR}$ and BABOK ${ }^{\circledR}$ functions applied within Printec Group-Software R \& D Division TMS support team have also been presented. Finally, the importance of defining services provision in an 'IT-enabled business' context has been discussed whereby the development and deployment of IT software products support corporate strategic envisioning, business vitality and viability through the achievement of specific business goals.

\section{Acknowledgements}

Special thanks to Printec Group of Companies and the Software R \& D Division which contributed to a significant degree to the research presented. Special thanks to
Dr. Fotis Moulatsiotis without whom the research would not have been realised. His impeccable judgment and proper guidance filled me with more determination to complete a thorough research. Many thanks to Alexander Poutos, Christos Michas and George Dimitropoulos for their contribution on IT service support matters and thanks to the POS Applications Development and POS Helpdesk teams of Printec Greece e-Payments Department. Many thanks also to the unknown referees. All contributions filled me with greater motivation and enthusiasm to conduct the research.

\section{REFERENCES}

[1] Office of Government Commerce, ITIL ${ }^{\circledR}$ v3-IT Service Management Core Books, TSO, 2007.

[2] Project Management Institute, "A Guide to the Project Management Body of Knowledge," 4th Edition, Project Management Institute, 2008.

[3] B. Hughes, R. Ireland, B. West, N. Smith and D. I. Shepherd, "Project Management for IT Related Projects," British Computer Society in Association with Biddles Ltd., London, 2004.

[4] IIBA, "A Guide to the Business Analysis Body of Knowledge (BABOK Guide)," International Institute of Business Analysis, 2009.

[5] P. Wheatcroft, "World Class IT Service Delivery," British Computer Society in Association with CAPDM, London, 2007.

[6] J. Robertson and S. Robertson, "Mastering the Requirements Process," 2nd Edition, Addison Wesley, Reading, 2006.

[7] E. Harrin, "Project Management in the Real World," British Computer Society in association with CAPDM 2007. 